Pacific Journal of Mathematics

POINT-DETERMINING HOMOMORPHISMS ON
MULTIPLICATIVE SEMI-GROUPS OF CONTINUOUS

ROBERT STEPHEN De Z 


\title{
POINT-DETERMINING HOMOMORPHISMS ON MULTIPLICATIVE SEMI-GROUPS OF CONTINUOUS FUNCTIONS
}

\author{
R. S. DEZur
}

Let $X$ and $Y$ be compact Hausdorff spaces, $C(X)$ and $C(Y)$ the algebras of real valued continuous functions on $X$ and $Y$ respectively with the usual sup norms. If $T$ is an algebra homomorphism from $C(X)$ onto a dense subset of $C(Y)$ then by a theorem of Stone, $T$ induces a homeomorphism $\mu$ from $Y$ to $X$ and it necessarily follows that $T f(y)=0$ if and only if $f(\mu(y))=0$.

In a more general setting, viewing $C(X)$ and $C(Y)$ as multiplicative semi-groups, let $T$ be a semi-group homomorphism from $C(X)$ onto a dense point-separating set in $C(Y)$. No such map $\mu$ satisfying the above condition need exist. $T$ is called point-determining in case for each $y$ there is an $x$ such that $T f(y)=0$ if and only if $f(x)=0$. It is shown that such a homomorphism $T$ induces a homeomorphism from $Y$ into $X$ in such a way that $T f(y)=[\operatorname{sgn} f(x)]|f(x)|^{p(x)}$ for some continuous positive function $p$ where $x$ is related to $y$ via the induced homeomorphism, that such a $T$ is an algebra homomorphism followed by a semi-group automorphism, and that $T$ is continuous.

Let $X$ and $Y$ be compact Hausdorff spaces, $C(X)$ and $C(Y)$ the algebras of all continuous real-valued functions on $X$ and $Y$ respectively with the usual sup norm. Let $T$ be an algebra homomorphism of $C(X)$ onto a dense set in $C(Y)$. For each $y \in Y$ consider the mapping $\gamma_{y}$ of $C(X)$ into the reals defined by

$$
\dot{\gamma}_{y}(f)=T f(y)
$$

$\gamma_{y}$ maps $C(X)$ onto the reals for if $T f(x)=0$ for all $f \in C(X)$ then the image of $T$ is not dense. The kernel is, by algebra, a maximal ideal in $C(X)$. By a theorem of Stone $[3,80]$ there is a point $x \in X$ so that the kernel of $\gamma_{y}$ is the set of all $f \in C(X)$ such that $f(x)=0$, this point being uniquely determined.

Consider the map $\mu$ of $Y$ into $X$ which assigns to each $y \in Y$ the $x$ as described above. If $e$ and $e_{1}$ are the unit functions in $C(X)$ and $C(Y)$ respectively it is easy to see that $T e=e_{1}$ and that $\mu$ is one-toone. Now for each $f \in C(X)$ consider the function $T f(y) e-f=g$ in $C(X)$. Then $T g(y)=0$ so that $g(\mu(y))=0$ and hence $T f(y)=f(\mu(y)$ ). We especially note that 


$$
T f(y)=0 \text { if and only if } f(\mu(y))=0 .
$$

As we shall see, under a more general setting for $T$, this condition will imply that $\mu$ is bicontinuous (see Lemma 3.1 below).

In this paper we view $C(X)$ and $C(Y)$ as multiplicative semi-groups and let $T$ be a semi-group homomorphism from $C(X)$ onto a dense set in $C(Y)$; the restriction on $T$ being that for each $y \in Y$ there is an $x \in X$ such that $f(x)=0$ if and only if $T f(y)=0$ (i.e. a condition such as (*) above is satisfied). For such a $T$ we show that $Y$ can be imbedded homeomorphically in $X$ in such a way that $T f(y)=[\operatorname{sgn} f(x)]|f(x)|^{p(x)}$ for some continuous positive function $p(x)$ where $y$ is related to $x$ via the induced homeomorphism. It is shown that each such homomorphism $T$ is an algebra homomorphism followed by a semi-group automorphism and that $T$ is continuous.

2. Definitions, Notation and Preliminaries. We first note that in our more general setting no mapping $\mu$ satisfying $\left(^{*}\right)$ above need exist. To see this let $X=[0,1] \cup\{2\}, Y=[0,1]$ with the relative topology of the real line. For $t \in[0,1]$ set

$$
T f(t)=f(t) f(2) \text {. }
$$

$T$ is a semi-group homomorphism of $C(X)$ onto $C(Y)$ but $T f(t)=0$ if and only if either $f(t)=0$ or $f(2)=0$.

Definition 2.1. A semi-group homomorphism $T$ will be called point-determining in case for each $y \in Y$ there is an $x \in X$ such that $f(x)=0$ if and only if $T f(y)=0$.

The following result is immediate.

LEMMA 2.2. If $T$ is a point-determining semi-group homomorphism of $C(X)$ onto a dense set in $C(Y)$, e and $e_{1}$ the respective unit functions in $C(X)$ and $C(Y)$ then $T e=e_{1}$ and $T O=O$.

Definition 2.3. A subset $A \subseteq C(Y)$ will be called point-separating in case for $y_{1} \neq y_{2}$ in $Y$ there is a $g \in A$ such that $g\left(y_{1}\right)=0$ and $g\left(y_{2}\right) \neq 0$.

In the development that follows $X$ and $Y$ will be compact Hausdorff spaces, $Y$ having no isolated points; $C(X)$ and $C(Y)$ will be viewed as multiplicative semi-groups and $T$ will be a point-determining semigroup homomorphism of $C(X)$ onto a dense point-separating set in $C(Y)$. The hypothesis $Y$ has no isolated points, however, is not used until Lemma 3.5. Multiplication is defined pointwise in $C(X)$ and $C(Y)$. $\sim A$ will denote complement of $A$ in any of the spaces considered. 
will denote the empty set and the bar notation will denote closure.

We are indebted to a paper of Milgram [2] for suggesting the sequence of ideas and devices employed here.

3. Development of the main results. Notice that for each $y \in Y, T$ determines a unique point $x \in X$. Thus $T$ induces a welldefined single valued mapping $\mu: Y \rightarrow X$ defined by $\mu(y)=x$ in case $f(x)=0$ if and only if $T f(y)=0$. In the material to follow notationally we let $\mu(Y)=X_{0}$. ( $\mu$ turns out to be a special case of the multivalued mappings studied in [1] although there we assumed $T$ continuous.)

Lemma 3.1. $\mu$ is a homeomorphism of $Y$ into $X$.

Proof. $\mu$ is a one-to-one for say $\mu\left(y_{1}\right)=\mu\left(y_{2}\right)$. Then $T f\left(y_{1}\right)=0$ if and only if $T f\left(y_{2}\right)=0$. If $y_{1} \neq y_{2}$ then since the range of $T$ is pointseparating there is an $h \in C(X)$ such that $T h\left(y_{1}\right)=0, T h\left(y_{2}\right) \neq 0$ a contradiction.

To see $\mu$ is continuous we suppose contrarywise that $\mu$ is not continuous at some point $t_{0} \in Y$. Then there is a net $\left\{t_{\beta}\right\}$ in $Y, t_{\beta} \rightarrow t_{0}$ and an open neighborhood $U$ containing $\mu\left(t_{0}\right)$ such that $\mu\left(t_{\beta}\right) \notin U$ for any $\beta$. Now there is an $f \in C(X)$ such that $f\left(\mu\left(t_{0}\right)\right)=1$ and $f(\sim U)=0$ so that $f\left(\mu\left(t_{\beta}\right)\right)=0$ for all $\beta$ and hence $T f\left(t_{\beta}\right)=0$ for all $\beta$. But $T f\left(t_{0}\right) \neq 0$ since $f\left(\mu\left(t_{0}\right)\right) \neq 0$ contradicting the fact that $T f \in C(Y)$. Thus $\mu$ is continuous and it follows that $\mu$ is a homeomorphism.

If $\sigma$ is a homeomorphism from $Y$ into $X$, define

$$
T: C(X) \rightarrow C(Y)
$$

by

$$
T f(y)=f(\sigma(y)), \quad f \in C(X), \quad y \in Y .
$$

$T$ is onto (and continuous) so that we have the following.

THEOREM 3.2. There is a point-determining semi-group homomorphism of $C(X)$ onto a dense point-separating set in $C(Y)$ if and only if $Y$ is homeomorphic to a subset of $X$.

We proceed now, to find the form of the general homomorphism in our theory.

Lemma 3.3. Let $U$ be open in $X$. If $f \equiv 1$ on $U$ then $T f \equiv 1$ on $\mu^{-1}\left(U \cap X_{0}\right)$. 
Proof. $f \equiv 1$ on $U$ implies that $f g=g$ for all $g \in C(X)$ such that $g(x)=0$ on $\sim U$ and hence $T f T g=T g$ for all such $g$. Let $y_{0} \in \mu^{-1}\left(U \cap X_{0}\right)$ so that $\mu\left(y_{0}\right)=x_{0} \in U \cap X_{0} \subset U$ and note that $T f\left(y_{0}\right) \neq 0$ since $f\left(x_{0}\right)=1$. Now there is an $h \in C(X)$ such that $h\left(x_{0}\right)=1$ and $h(\sim U)=0$ so from the above $T f\left(y_{0}\right) T h\left(y_{0}\right)=T h\left(y_{0}\right)$. But $h\left(x_{0}\right)=1$ implies that $T h\left(y_{0}\right) \neq 0$ and therefore $T f\left(y_{0}\right)=1$ and since $y_{0}$ was arbitrary the result follows.

Lemma 3.4. Let $U$ be open in $X$. If $f \equiv g$ on $U$ then $T f \equiv T g$ on $\mu^{-1}\left(U \cap X_{0}\right)$.

Proof. Let $y_{0} \in \mu^{-1}\left(U \cap X_{0}\right)$ so that $\mu\left(y_{0}\right)=x_{0} \in U \cap X_{0} \subset U$. If $f\left(x_{0}\right)=0=g\left(x_{0}\right)$ then $T f\left(y_{0}\right)=0=T g\left(y_{0}\right)$. If $f\left(x_{0}\right)=g\left(x_{0}\right) \neq 0$ we may assume without loss of generality that $f\left(x_{0}\right)=g\left(x_{0}\right)=c>0$. Then $W^{\prime}=\{x \mid f(x)>c / 2\}$ is open in $X$ and $x_{0} \in W^{\prime}$. For $x \in X$ set $h^{\prime}(x)=$ $\max [f(x), c / 2]$. Then $h^{\prime}$ and $h=1 / h^{\prime}$, are in $C(X)$. Now $f h \equiv 1$ on $W^{\prime}$ and hence $f h \equiv 1 \equiv g h$ on $W=W^{\prime} \cap U$. Thus by Lemma 3.3 $T f h \equiv 1 \equiv T g h$ on $\mu^{-1}\left(W \cap X_{0}\right)$ and so in particular $T f\left(y_{0}\right) T h\left(y_{0}\right)=1=$ $T g\left(y_{0}\right) T h\left(y_{0}\right)$. Now $h\left(x_{0}\right) \neq 0$ so $T h\left(y_{0}\right) \neq 0$. Thus $T f\left(y_{0}\right)=T g\left(y_{0}\right)$ and the result follows.

LEMma 3.5. Let $x_{0}=\mu\left(y_{0}\right)$. If $f\left(x_{0}\right)=1$ then $T f\left(y_{0}\right)=1$.

Proof. Suppose first that $f\left(x_{0}\right)=1$ but that $T f\left(y_{0}\right)>1$. Then there is an open neighborhood $W$ containing $y_{0}$ such that $T f\left(y_{0}\right) \geqq c>1$ for all $y \in W$. Now $\mu(W)=U \cap X_{0}$ for some open set $U$ in $X$ such that $x_{0} \in U$. Let $V_{n}=\left\{x \in X|| f^{n}(x)-1 \mid<1 / n\right\} \quad n=1,2,3, \cdots$ and set $U_{n}=V_{n} \cap U$. Note that $x_{0} \in U_{n}$ an open set in $X$ for each $n$ and that there are points of $X_{0}-\left\{x_{0}\right\}$ in $U_{n}$ for every $n$ since $Y$ has no isolated points (and hence $X_{0}$ has none). We construct a sequence $\left\{x_{n}\right\}$ of distinct points such that $x_{n} \in U_{n} \cap X_{0}$ as follows:

Select $x_{1} \in U_{1} \cap X_{0}$ such that $x_{1} \neq x_{0}$ and set $U_{1}=W_{0}^{(0)}$. Select disjoint neighborhoods $W_{0}^{(1)}$ containing $x_{0}$ and $W_{1}^{(1)}$ containing $x_{1}$ such that $W_{0}^{(1)} \subset W_{0}^{(0)}$ and $W_{1}^{(1)} \subset W_{0}^{(0)}$.

In general select $x_{n} \in W_{0}^{(n-1)} \cap X_{0} \cap U_{n}$ such that $x_{n} \neq x_{0}$ and disjoint neighborhoods $W_{0}^{(n)}$ containing $x_{0}$ and $W_{1}^{(n)}$ containing $x_{n}$ such that $W_{0}^{(n)} \subset W_{0}^{(n-1)}$ and $W_{1}^{(n)} \subset W_{0}^{(n-1)}$. Note that $\left\{W_{0}^{(n)}\right\}$ is a decreasing sequence of neighborhoods containing $x_{0}$ where $x_{i} \in W_{0}^{(i-1)} ;\left\{W_{1}^{(n)}\right\}$ is a collection of neighborhoods where $x_{i} \in W_{1}^{(i)}$ and $W_{1}^{(n)} \cap W_{0}^{(n)}=\varnothing$ $n=1,2,3, \cdots$.

For the sequence $\left\{x_{n}\right\}$ we have $\left\{x_{n+1}, x_{n+2}, \cdots\right\} \subset W_{0}^{(n)}$ and $W_{1}^{(n)}$ is a neighborhood containing $x_{n}$ such that $\left\{x_{n+1}, x_{n+2}, \cdots\right\} \cap W_{1}^{(n)}=\varnothing$. Therefore $x_{n} \notin\left\{\overline{x_{n+1}, x_{n+2}, \cdots}\right\}$. Hence we can select a collection $\left\{O_{n}\right\}$ of open sets as follows: 
Let $O_{1}$ be an open subset of $U_{1}$ such that $x_{1} \in O_{1} \subset \bar{O}_{1} \subset U_{1}$ and $\bar{O}_{1}$ does not contain $x_{2}, x_{3}, \cdots$. In general let $O_{n}$ be an open subset of $U_{n}$ such that $x_{n} \in O_{n} \subset \bar{O}_{n} \subset U_{n}$ and $\bar{O}_{n}$ does not contain $x_{n+1}, x_{n+2}, \cdots$ and such that $\bar{O}_{i} \cap \bar{O}_{n}=\varnothing i=1,2, \cdots, n-1$.

Now define a function $g^{\prime}$ on $\overline{\bigcup_{n=1}^{\infty} \bar{O}_{n}}$ by

$$
g^{\prime}=\left\{\begin{array}{l}
f^{n} \text { on } \bar{O}_{n} \\
1 \text { elsewhere on } \overline{\bigcup_{n=1}^{\infty} \bar{O}_{n}} .
\end{array}\right.
$$

Then $g^{\prime}$ is continuous on $\overline{\mathrm{U}}_{n=1}^{\infty} \bar{O}_{n}$. To see this we need only examine

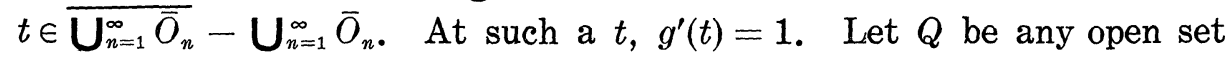
in the reals containing 1 and choose $N>0$ such that $(1-1 / N, 1+$ $1 / N) \subset Q$. Now since $t \notin \bigcup_{i=1}^{N} \bar{O}_{i}$, a closed set, there is a neighborhood $V$ containing $t$ such that $V \cap \bigcup_{i=1}^{N} \bar{O}_{i}=\varnothing$. For $s \in V \cap{\overline{\bigcup_{i=N+1}^{\infty}}}_{i}$ either $g^{\prime}(s)=1$ or $s \in \bar{O}_{k}$ for some $k \in\{N+1, N+2, \cdots\}$ in which case $\left|g^{\prime}(s)=1\right|=\left|f^{k}(s)-1\right|<1 / k<1 / N$. So in any case $\left|g^{\prime}(s)-1\right|<1 / N$ i.e. $g^{\prime}(s) \in Q$ and hence $g^{\prime}$ is continuous. By Tietze's extension theorem $g^{\prime}$ can be extended to a function $g \in C(X)$.

Now for $y \in \mu^{-1}\left(O_{n} \cap X_{0}\right) \subset W$ we have by Lemma 3.4

$$
T g(y)=T f^{n}(y)=[T f(y)]^{n} \geqq c^{n}
$$

so $T g$ is not bounded and hence $T g \notin C(Y)$ a contradiction. Thus if $f\left(x_{0}\right)=1$ then $T f\left(y_{0}\right) \leqq 1$.

Now suppose that $f\left(x_{0}\right)=1$ but that $T f\left(y_{0}\right)<1$. Set $f_{1}=f^{2}$. Then $f_{1}\left(x_{0}\right)=1$ so $T f_{1}\left(y_{0}\right) \neq 0$ and $T f_{1}\left(y_{0}\right)=\left[T f\left(y_{0}\right)\right]^{2}>0$. By the first part of the proof $T f_{1}\left(y_{0}\right) \ngtr 1$. We rule out $T f_{1}\left(y_{0}\right)<1$ as follows.

Set $W=\left\{x \mid f_{1}(x)>1 / 2\right\} . \quad W$ is open, $x_{0} \in W$ and if we set $f_{2}(x)=$ $\max \left[1 / 2, f_{1}(x)\right]$ then $f_{2}$ is nowhere zero, $f_{2} \in C(X)$ and $f_{2}$ agrees with $f_{1}$ on $W$. Hence by Lemma $3.4 T f_{1} \equiv T f_{2}$ on $\mu^{-1}\left(W \cap X_{0}\right)$ and so $0<T f_{2}\left(y_{0}\right)<1$. Now $f_{3}=1 / f^{2} \in C(X), f_{3}\left(x_{0}\right)=1$ and $T f_{3}\left(y_{0}\right)=1 / T f_{2}\left(y_{0}\right)>1$ a contradiction by the first part of the proof. Hence $T f_{1}\left(y_{0}\right)=1$ so that $T f\left(y_{0}\right)= \pm 1$. But by assumption $T f\left(y_{0}\right)<1$ so $T f\left(y_{0}\right)=-1$.

As done above let $g$ be a strictly positive function in $C(X)$ agreeing with $f$ on some neighborhood $U$ containing $x_{0}$. Then Tf and Tg agree on $\mu^{-1}\left(U \cap X_{0}\right)$. But $g>0$ everywhere on $X$ implies that $T g \geqq 0$ everywhere on $Y$ and hence $T g\left(y_{0}\right) \neq-1$ so $T f\left(y_{0}\right) \neq-1$, a contradiction. Thus $T f\left(y_{0}\right)=+1$ and the proof is finished.

LEMmA 3.6. If $x_{0}=\mu\left(y_{0}\right)$ and if $f\left(x_{0}\right)=g\left(x_{0}\right)$ then $T f\left(y_{0}\right)=T g\left(y_{0}\right)$.

Proof. We need only consider $f\left(x_{0}\right)=c=g\left(x_{0}\right) \neq 0$. Let $h(x)=1 / c$ for all $x \in X$ so that $h \in C(X)$ and $h f\left(x_{0}\right)=h g\left(x_{0}\right)=1$. By Lemma 3.5 $T h f\left(y_{0}\right)=1=T h g\left(y_{0}\right)$ i.e. $T h\left(y_{0}\right) T f\left(y_{0}\right)=T h\left(y_{0}\right) T g\left(y_{0}\right)=1$. But $T h\left(y_{0}\right) \neq 0$ 
so the result follows.

Notice that Lemma 3.6 implies us that functions in $C(X)$ which agree on $X_{0}=\mu(Y)$ have the same images in $C(Y)$. We will show that $T$ is actually restriction to $X_{0}$ followed by a semi-group automorphism.

Suppose we regard the real numbers, $R$, as a multiplicative semigroup. We have the following.

LEMMA 3.7. Let $\alpha$ be a semi-group homomorphism from $R$ onto a dense subset of $R$. Then $\alpha$ is either unbounded in every neighborhood of zero or $\alpha$ is order preserving.

Proof. Since the range of $\alpha$ is dense in $R$ it follows that $\alpha(0)=0$ and $\alpha(1)=1$. If we show that $\alpha(-t)=-\alpha(t)$ for all $t$ then only positive numbers need be considered in verifying the lemma. To this end note $\alpha(1)=[\alpha(-1)]^{2}$ so $\alpha(-1)= \pm 1$. We rule out $\alpha(-1)=+1$ for suppose $\alpha(-1)=+1$. Then $\alpha( \pm t)=\alpha(t)$ for all $t$. Let $\left\{t_{n}\right\}$ be a sequence in $R$ such that $\alpha\left(t_{n}\right) \rightarrow-1$. Then $\alpha\left(-t_{n}\right)=\alpha\left(t_{n}\right) \rightarrow-1$ so that $\alpha\left(\left|t_{n}\right|\right) \rightarrow-1$. But $\left|t_{n}\right|=s_{n}^{2}$ for some $s_{n} \in R$ and $\alpha\left(s_{n}^{2}\right)=\left[\alpha\left(s_{n}\right)\right]^{2} \rightarrow$ -1 a contradiction. Hence $\alpha(-t)=-\alpha(t)$.

Now let $a, b \in R$ such that $0<a<b$. Suppose $\alpha(a)>\alpha(b)$. Then since $\alpha(a / b)=\alpha(a) / \alpha(b)$ we have $\alpha\left[(a / b)^{n}\right]=[\alpha(a) / \alpha(b)]^{n} \rightarrow \infty$ while $(a / b) \rightarrow 0$ i.e. $\alpha$ is unbounded in every neighborhood of zero. Now suppose $\alpha(a)=\alpha(b)$ and that $\alpha$ is bounded in some neighborhood of zero. Then $r \in[a, b]$ implies that $\alpha(r)=\alpha(a)$ since otherwise either $\alpha(r)<\alpha(a)$ or $\alpha(r)>\alpha(b)$ and in both cases by the above $\alpha$ would be unbounded in every neighborhood of zero contradicting our assumption. Hence for $r, r^{\prime} \in[a, b] \alpha\left(r / r^{\prime}\right)=\alpha(r) / \alpha\left(r^{\prime}\right)=1$. Now let $z$ be any positive real number. There is an $n$ such that $a / b \leqq z^{1 / n} \leqq b / a$ i.e. there is an $n$ such that $z^{1 / n}=r / r^{\prime}$, where $r, r^{\prime} \in[a, b]$. Then $1=\alpha\left(z^{1 / n}\right)$ and so $\alpha(z)=\left[\alpha\left(z^{1 / n}\right)\right]^{n}=1$. So $z>0$ implies that $\alpha(z)=1, z<0$ implies that $\alpha(z)=-1$ and $\alpha(0)=0$, a contradiction since the image of $\alpha$ is dense in $R$. Thus $\alpha(a)=\alpha(b)$ implies that $\alpha$ is unbounded in every neighborhood of zero.

LEMMA 3.8. If $\alpha$ is order preserving then $\alpha$ is actually onto and in this case $\alpha(t)=(\operatorname{sgn} t)|t|^{p}$ for some positive number $p$.

Proof. Let $r_{0} \in R$ and $\left\{r_{n}\right\}$ a sequence in $R$ such that $\left\{r_{n}\right\} \downarrow r_{0}$. Then $\alpha\left(r_{n}\right) \rightarrow \alpha\left(r_{0}\right)$ since $\alpha\left(r_{n}\right)>\alpha\left(r_{0}\right)$ and if $\alpha\left(r_{n}\right) \geqq m>\alpha\left(r_{0}\right)$ there is an $s \in R, m>s>\alpha\left(r_{0}\right)$ and a $q \in R$ such that $\alpha(q)=s$. But $\alpha\left(r_{0}\right)<\alpha(q)<\alpha\left(r_{n}\right)$ for all $n$ so $r_{0}<q<r_{n}$, a contradiction since $r_{n} \rightarrow r_{0}$. 
To see $\alpha$ is onto say $r_{0}$ is such that $\alpha(r) \neq r_{0}$ for any $r \in R$. We can choose a sequence of distinct points $\left\{\alpha\left(r_{n}\right)\right\}$ such that $\alpha\left(r_{n}\right) \downarrow r_{0}$. This implies $\left\{r_{n}\right\}$ is a bounded decreasing sequence so there is an $r^{\prime}$ such that $r_{n} \downarrow r^{\prime}$ and hence from the above $\alpha\left(r_{n}\right) \rightarrow \alpha\left(r^{\prime}\right)$, a contradiction since $\alpha\left(r^{\prime}\right) \neq r_{0}$. Thus $\alpha$ is onto. Milgram [2, 4.3] has shown that in this case there is a $p>0$ such that $\alpha(t)=(\operatorname{sgn} t)|t|^{p}$ which completes the proof.

In view of Lemma 3.6 for each $y \in Y, \alpha_{\mu(y)}: R \rightarrow R$ defined for arbitrary $f \in C(X)$ by $\alpha_{\mu(y)}(f(\mu(y)))=T f(y)$ is well-defined. The image of $\alpha_{\mu(y)}$ is a dense subset in $R$, for fix $y \in Y$ and let $r \in R$. There is a function $g \in C(Y)$ such that $g(y)=r$ and a sequence $\left\{f_{n}\right\} \subset C(X)$ such that $T f_{n}(y) \rightarrow g(y)=r$ i.e. $\alpha_{\mu(y)}\left(f_{n}(\mu(y))\right) \rightarrow r$.

Note that from Lemmas 3.7 and 3.8 we can say that $\alpha_{\mu(y)}$ is unbounded in every neighborhood of zero or $\alpha_{\mu(y)}$ is continuous.

LEMMA 3.9. The mappings $\left\{\alpha_{\mu(y)}\right\}$ are discontinuous for at most a finite number of points.

Proof. Suppose otherwise at $\left\{\mu\left(y_{n}^{\prime}\right)\right\}$ where the $y_{n}^{\prime}$ are all distinct $n=1,2,3, \cdots$. We can choose a subsequence $\left\{\mu\left(y_{n}\right)\right\}$ of distinct points such that no $\mu\left(y_{n}\right)$ is a limit point of the others as follows:

If no point in $\left\{\mu\left(y_{n}^{\prime}\right)\right\}$ is a limit point of the other we are finished. If $y_{n_{0}}^{\prime}$ is a limit point of a subset of $\left\{\mu\left(y_{n}^{\prime}\right)\right\}$ where $y_{n_{0}}^{\prime} \in\left\{\mu\left(y_{n}^{\prime}\right)\right\}$, by a process similar to that used in selecting the sequence $\left\{x_{n}\right\}$ in the proof of Lemma 3.5 with $y_{n_{0}}^{\prime}$ in the role of $x_{0}$ we obtain a sequence $\left\{\mu\left(y_{n}\right)\right\}$ such that $\mu\left(y_{n}\right) \notin\left\{\overline{\mu\left(y_{n+1}\right), \mu\left(y_{n+2}\right), \cdots}\right\}, n=1,2,3, \cdots$. Hence for any $\mu\left(y_{n}\right)$ there is an open set $V$ containing $\mu\left(y_{n}\right)$ such that $V \cap\left\{\mu\left(y_{n}\right)\right\}-$ $\mu\left(y_{n}\right)=\varnothing$ so that $\left\{\mu\left(y_{n}\right)\right\}$ is the desired collection.

Now the $\alpha_{\mu\left(y_{n}\right)}$ are unbounded in each neighborhood of the origin so that if $\left\{t_{m}^{\prime}\right\}$ is a sequence of distinct points decreasing to zero we have $\alpha_{\mu\left(y_{n}\right)}\left(t_{m}^{\prime}\right) \rightarrow \infty$ for all $n$ as $m \rightarrow \infty$. We select a subsequence $\left\{t_{n}\right\} \downarrow 0$ such that $\alpha_{\mu\left(y_{n}\right)}\left(t_{n}\right) \rightarrow \infty$ as follows:

There is a $t \in\left\{t_{m}^{\prime}\right\}$ such that $\alpha_{\mu\left(y_{1}\right)}(t)>1$. Set $t=t_{1}$. In general there is a $t<t_{n-1}<\cdots<t_{1}, t \in\left\{t_{m}^{\prime}\right\}$ such that $\alpha_{m\left(y_{n}\right)}(t)>n$. Set $t=t_{n}$ to yield the desired sequence.

Define a function $f^{\prime}$ on $\left\{\overline{\mu\left(y_{n}\right)}\right\}$ by $f^{\prime}\left(\mu\left(y_{n}\right)\right)=t_{n}$ and $f^{\prime}=0$ on $\left\{\overline{\mu\left(y_{n}\right)}\right\}-\left\{\mu\left(y_{n}\right)\right\} . \quad f^{\prime}$ is continuous on $\left\{\overline{\mu\left(y_{n}\right)}\right\}$ since for $y_{0} \in\left\{\overline{\mu\left(y_{n}\right)}\right\}-$ $\left\{\mu\left(y_{n}\right)\right\}$ we have $f^{\prime}\left(y_{0}\right)=0$ and letting $\left\{\mu\left(y_{m}\right)\right\}$ be any subsequence converging to $y_{0}, f^{\prime}\left(\mu\left(y_{m}\right)\right)=t_{m} \rightarrow 0=f^{\prime}\left(y_{0}\right)$.

Now we can extend $f^{\prime}$ to a continuous function $f$ on all of $X$. But then $T f\left(y_{n}\right)=\alpha_{\mu\left(y_{n}\right)}\left(f\left(\mu\left(y_{n}\right)\right)\right)=\alpha_{\mu\left(y_{n}\right)}\left(t_{n}\right) \rightarrow \infty$ contradicting the fact that $T f \in C(Y)$ and the lemma is proved. 
We have via Lemma 3.8, that except for at most a finite number of points $y$,

$$
\alpha_{\mu(y)} f(\mu(y))=[\operatorname{sgn} f(\mu(y))]|f(\mu(y))|^{p(\mu(y))} \quad \text { where } p(\mu(y))
$$

is a positive function. We note that $p$ is continuous where it is defined, i.e. on the set $\left\{\mu(y) \mid \alpha_{\mu(y)}\right.$ is continuous $\}$, since for the constant function 2 we have $T 2(y)=\alpha_{\mu(y)}(2)=[\operatorname{sgn} 2]|2|^{p(\mu(y))}=2^{p(\mu(y))}$ and since T2 is continuous the result follows.

Using the fact that $Y$ has no isolated points we show a stronger result.

Lemma 3.10. There is a positive continuous function $p$ on $X_{0}$ such that

$$
\alpha_{\mu(y)}(f(\mu(y)))=[\operatorname{sgn} f(\mu(y))]|f(\mu(y))|^{p(\mu(y))} .
$$

Proof. In view of the preceeding remarks we need only show that $\alpha_{\mu(y)}$ is continuous for all $y$. To this end suppose that $\alpha_{\mu(y)}$ is discontinuous at $y_{0}$. Set $A=\left\{\mu(y) \mid \alpha_{\mu(y)}\right.$ is continuous $\}$. By Lemma 3.9 all but a finite number of the $\mu(y)$ are in $A$ and hence since $Y$ has no isolated points every open neighborhood about $\mu\left(y_{0}\right)$ contains points of $A$.

Now for $0<s<1$ define $S \in C(X)$ by $S(x)=s$. Since $\alpha_{\mu\left(y_{0}\right)}$ is unbounded in every neighborhood of zero we can find an $s_{0} \in(0,1)$ such that $\alpha_{\mu\left(y_{0}\right)}\left(s_{0}\right)>2$. Let $U$ be any neighborhood containing $\mu\left(y_{0}\right)$ and take $\mu(y) \in U \cap A$. Then $T S_{0}(y)=\alpha_{\mu(y)}\left(s_{0}\right)=\left[\operatorname{sgn} s_{0}\right]\left|s_{0}\right|^{p(\mu(y))}<1$ but $T S_{0}\left(y_{0}\right)=\alpha_{\mu\left(y_{0}\right)}\left(s_{0}\right)>2$ which contradicts the continuity of $T S_{0}$.

LEMMA 3.11. The semi-group homomorphism $T$ is an algebra homomorphism followed by a semi-group automorphism. Moreover $T$ is continuous.

Proof. From Lemma 3.10 we have

$$
T f(y)=[\operatorname{sgn} f(\mu(y))]|f(\mu(y))|^{p(\mu(y))} .
$$

Identify $Y$ as the subset $X_{0}$ of $X$ and define $T_{1}: C(X) \rightarrow C(Y)$ by $T_{1} f=$ $f \mid Y$ (i.e. $f$ restricted to $Y$ ) and note that $T_{1}$ is an onto algebra homomorphism. Define $T_{2}: C(Y) \rightarrow C(Y)$ by $T_{2} g(y)=[\operatorname{sgn} g(y)]|g(y)|^{p(y)}$ where $p(y)$ is the continuous positive function arising in the previous lemma. $\quad T_{2}$ is a semi-group automorphism. To see that $T_{2}$ is one-toone suppose $f_{1}, f_{2} \in C(Y)$ where $f_{1} \neq f_{2}$. Then there is a $y \in Y$ such that $f_{1}(y) \neq f_{2}(y)$. Now if $\left|f_{1}(y)\right| \neq\left|f_{2}(y)\right|$ then $T_{2} f_{1}(y) \neq T_{2} f_{2}(y)$ and if $\left|f_{1}(y)\right|=\left|f_{2}(y)\right|$ then $\operatorname{sgn} f_{1}(y) \neq \operatorname{sgn} f_{2}(y)$ so that $T_{2} f_{1}(y) \neq T_{2} f_{2}(y)$. Thus $T_{2}$ is one-to-one. Clearly $T=T_{2} T_{1}$. 
To see that $T$ is continuous it suffices to show that $T_{2}$ is continuous ( $T_{1}$ is clearly continuous). A standard argument shows this to be the case.

Combining some of the previous results we have the following.

THEOREM 3.12. Let $X$ and $Y$ be compact Hausdorff spaces, $Y$ having no isolated points. Let $C(X)$ and $C(Y)$ be the multiplicative semi-groups of all continuous real valued function on $X$ and $Y$ respectively. If $T$ is a point-determining semi-group homomorphism of $C(X)$ onto a dense point-separating set in $C(Y)$ then $Y$ can be imbedded homeomorphically in $X$ in such a way that

$$
T f(y)=[\operatorname{sgn} f(x)]|f(x)|^{p(x)}
$$

for some continuous positive function $p$ where $x$ is the unique point related to $y$ by the induced homeomorphism. Such a homomorphism is continuous and is an algebra homomorphism followed by a semigroup automorphism.

Corollary 3.13. Let $X$ and $Y$ be compact Hausdorff spaces, $Y$ having no isolated points. Let $T$ be a semi-group homomorphism of $C(X)$ onto a dense point-separating set of $C(Y)$. Then

(i) $T$ is an algebra homomorphism of $C(X)$ into $C(Y)$ if and only if $T$ is point-determining and $T c=c$ for each constant function $c$.

(ii) If $T$ is point-determining then $T(-f)=-T f$.

Proof. (i) If $T$ is an algebra homomorphism of $C(X)$ we have already seen that $T$ is point-determining and in fact that $T f(y)=f(\mu(y))$ where $\mu$ is the induced homeomorphism. Hence $T c=c$ for all constant functions $c$.

If $T$ is point-determining and $T c=c$ for all constant functions $c$ then by the above theorem, for all $y$,

$$
2=T_{2}(y)=[\operatorname{sgn} 2] 2^{p(\mu(y))}=2^{p(\mu(y))}
$$

and hence $p(\mu(y))=1$ for all $y$. Thus for $f \in C(X), T f(y)=f(\mu(y))$ so $T$ is an algebra homomorphism.

The proof of (ii) is obvious by the form of the homomorphism shown in the above theorem.

\section{BIBLIOGRAPHY}

1. R. S. DeZur, Homomorphisms on multiplicative semi-groups of continuous functions on a compact space (to appear). 
2. A. N. Milgram, Multiplicative semi-groups of continuous functions, Duke Math. J. 16 (1949), 377-383.

3. M. H. Stone, Applications of the theory of Boolean rings to general topology, Trans. Amer. Math. Soc. 41 (1937), 375-481.

Received October 4, 1964.

RIAS

and

SANDIA CORPORATION-LIVERMORE LABORATORY 


\section{PACIFIC JOURNAL OF MATHEMATICS}

\section{EDITORS}

H. SAMELSON

Stanford University

Stanford, California

R. M. BLUMENTHAL

University of Washington

Seattle, Washington 98105

\section{*J. DugundJI}

University of Southern California Los Angeles, California 90007

RICHARD ARENS

University of California

Los Angeles, California 90024

\section{ASSOCIATE EDITORS}

E. F. BECKENBACH
F. WOLF

K. YosIDA

\section{SUPPORTING INSTITUTIONS}

\author{
UNIVERSITY OF BRITISH COLUMBIA \\ CALIFORNIA INSTITUTE OF TECHNOLOGY \\ UNIVERSITY OF CALIFORNIA \\ MONTANA STATE UNIVERSITY \\ UNIVERSITY OF NEVADA \\ NEW MEXICO STATE UNIVERSITY \\ OREGON STATE UNIVERSITY \\ UNIVERSITY OF OREGON \\ OSAKA UNIVERSITY \\ UNIVERSITY OF SOUTHERN CALIFORNIA
}

\author{
STANFORD UNIVERSITY \\ UNIVERSITY OF TOKYO \\ UNIVERSITY OF UTAH \\ WASHINGTON STATE UNIVERSITY \\ UNIVERSITY OF WASHINGTON \\ AMERICAN MATHEMATICAL SOCIETY \\ CHEVRON RESEARCH CORPORATION \\ TRW SYSTEMS \\ NAVAL ORDNANCE TEST STATION
}

Mathematical papers intended for publication in the Pacific Journal of Mathematics should be typewritten (double spaced). The first paragraph or two must be capable of being used separately as a synopsis of the entire paper. It should not contain references to the bibliography. Manuscripts may be sent to any one of the four editors. All other communications to the editors should be addressed to the managing editor, Richard Arens at the University of California, Los Angeles, California 90024 .

50 reprints per author of each article are furnished free of charge; additional copies may be obtained at cost in multiples of 50 .

The Pacific Journal of Mathematics is published monthly. Effective with Volume 16 the price per volume (3 numbers) is $\$ 8.00$; single issues, $\$ 3.00$. Special price for current issues to individual faculty members of supporting institutions and to individual members of the American Mathematical Society: $\$ 4.00$ per volume; single issues $\$ 1.50$. Back numbers are available.

Subscriptions, orders for back numbers, and changes of address should be sent to Pacific Journal of Mathematics, 103 Highland Boulevard, Berkeley 8, California.

Printed at Kokusai Bunken Insatsusha (International Academic Printing Co., Ltd.), No. 6, 2-chome, Fujimi-cho, Chiyoda-ku, Tokyo, Japan.

PUBLISHED BY PACIFIC JOURNAL OF MATHEMATICS, A NON-PROFIT CORPORATION

The Supporting Institutions listed above contribute to the cost of publication of this Journal, but they are not owners or publishers and have no responsibility for its content or policies.

* Paul A. White, Acting Editor until J. Dugundji returns. 


\section{Pacific Journal of Mathematics}

\section{Vol. 18, No. 2 \\ April, 1966}

Alexander V. Arhangelskii, On closed mappings, bicompact spaces, and a problem of P. Aleksandrov .............................. 201

A. K. Austin, A note on loops . . . . . . . . . . . . . . . . . . . . . . . . . . . . 209

Lawrence Peter Belluce and William A. Kirk, Fixed-point theorems for families of contraction mappings...................... 213

Luther Elic Claborn, Every abelian group is a class group ............ 219

Luther Elic Claborn, A note on the class group .................. 223

Robert Stephen De Zur, Point-determining homomorphisms on multiplicative semi-groups of continuous functions............. 227

Raymond William Freese, A convexity property ................ 237

Frederick Paul Greenleaf, Characterization of group algebras in terms of their translation operators ......................... 243

Andrzej Hulanicki, On the spectral radius of hermitian elements in group algebras....................................... 277

Michael Bahir Maschler and Bezalel Peleg, A characterization, existence proof and dimension bounds for the kernel of a game ............ 289

Yiannis (John) Nicolas Moschovakis, Many-one degrees of the predicates

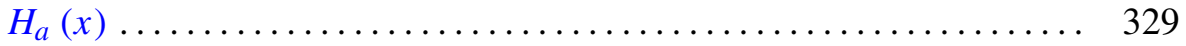

G. O. Okikiolu, $n$th order integral operators associated with Hilbert transforms.

C. E. Rickart, Analytic phenomena in general function algebras ... 361

K. N. Srivastava, On an entire function of an entire function defined by Dirichlet series

Paul Elvis Waltman, Oscillation criteria for third order nonlinear differential equations .......................... 